\title{
Estrategia de transformación digital para fortalecer el desempeño docente
}

Fecha de recepción: 2021-08-02 • Fecha de aceptación: 2021-10-15 • Fecha de publicación: 2021-12-01

Maya Aracely Sánchez Soto ${ }^{1}$

Universidad Técnica De Babahoyo, Ecuador

sanchezmaya9@gmail.com

http://Orcid.org/0000-0001-5929-6326

Gina Lorena Camacho Tovar ${ }^{2}$ Universidad Técnica De Babahoyo, Ecuador

CORREO:gtovar@utb.edu.ec http://Orcid.org/0000-0002-9824-2141

Edgar Stalyn Guerrero Haro ${ }^{3}$ Universidad Técnica De Babahoyo, Ecuador eguerrero@utb.edu.ec http://Orcid.org/0000-0003-0584-2410

Cruz Maribel Galarza Ramírez ${ }^{4}$ Universidad Técnica De Babahoyo, Ecuador cgalarzar@utb.edu.ec http://Orcid.org/0000-0001-9189-3294

\section{Resumen}

El estudio tuvo como objetivo proponer estrategias de transformación digital para fortalecer el desempeño docente con el fin de establecer beneficios de su uso en los procesos educativos. La metodología aplicada fue de enfoque mixto; considerando el tipo de investigación fue un estudio aplicado, por el alcance descriptivo y explicativo. La población de estudio estuvo conformada por 312 miembros de la Universidad Técnica de Babahoyo, Ecuador, incluidos docentes y estudiantes, 
la muestra se determinó por un muestro no probabilístico por conveniencia, en donde se trabajó con 172 sujetos y los datos se recolectaron por medio de la encuesta. Entre los principales resultados se obtuvo que la transformación digital y la revolución digital tuvieron un valor significativo de 0.000 , esto demuestra que la transformación digital se relaciona de forma considerable con el desempeño docente. Se concluye que las estrategias transformación digital mediante el uso de tecnologías como el Internet, los recursos y los avances tecnológicos en la era digital facilitan los procesos de aprendizajes, haciéndolos más activos y al relacionarse significativamente con el desempeño del docente benefician los procesos educativos con la mejora el flujo de información.

Palabras clave: desempeño docente, estrategia de transformación, procesos educativos, revolución tecnológica, transformación digital

\section{Abstract}

The objective of the study was to propose digital transformation strategies to strengthen teaching performance in order to establish the benefits of its use in educational processes. The methodology applied was of mixed approach; considering the type of research it was an applied study, due to its descriptive and explanatory scope. The study population consisted of 312 members of the Technical University of Babahoyo, Ecuador, including teachers and students, the sample was determined by a non-probabilistic convenience sample, where 172 subjects were worked with and data were collected by means of a survey. Among the main results it was obtained that digital transformation and digital revolution had a significant value of 0.000 , this shows that digital transformation is significantly related to teaching performance. It is concluded that digital transformation strategies through the use of technologies such as the Internet, resources and technological advances in the digital era facilitate learning processes, making them more active and significantly related to teacher performance, benefiting educational processes by improving the flow of information.

Keywords: teaching performance, transformation strategy, educational processes, technological revolution, digital transformation 


\section{Introducción}

En el ámbito educativo, a nivel global se ha evidenciado que existe la aceptación o el rechazo de la fase educativa, lo cual se concierne a las capacidades del docente y al ámbito social, cultural y económico. Escribano (2018) manifiesta que existen factores que están relacionados a la educación y a la calidad de la misma, es notorio que, el profesor tiene grandes desafíos al brindar una educación de calidad acorde a las exigencias del siglo XXI.

Bonilla et al. (2020) exponen que las universidades han reconocido la necesidad de mejorar la calidad de enseñanza, enfatizando la relevancia que tiene el considerar el criterio del alumnado sobre la gestión docente. En tal sentido, el profesor puede ser analizado para identificar debilidades, o por lo contrario, dentro del ámbito educativo (Martínez et al., 2016). Un docente con cualidades para impartir catedra es una persona que cuenta con las capacidades y cualidades académicas requeridas para enseñar en función a las políticas y niveles (Zamora et al., 2017).

En consideración al desempeño del docente, estas tienen que ver con las acciones relacionadas con el estudiante y se aplica en contextos tanto como el institucional, social, y el aula, elemento que en la actualidad es considerado relevante para obtener resultados importantes y positivos en el alumnado (Folch et al., 2018). A medida que el conocimiento sobre el desarrollo y el aprendizaje humano ha crecido a un ritmo rápido, también ha aumentado la oportunidad de dar forma a prácticas educativas más eficaces. Los desafíos para la educación en el siglo XXI son descubrir y desarrollar herramientas que agreguen valor tanto a la enseñanza, como al aprendizaje. El panorama cambiante de la educación superior se ve afectado significativamente por las nuevas tecnologías y los cambios en la demografía de los estudiantes (Sánchez, 2020).

De acuerdo a lo anterior anterior, para ayudar a los estudiantes a alcanzar sus expectativas, las instituciones y los docentes compiten para brindar un apoyo que se adapte a las necesidades de estos diversos grupos. Minero (2020) menciona que emplear diferentes estrategias y métodos de enseñanza bajo una modalidad en línea, implica que los docentes incrementen una participación más proactiva por parte de los estudiantes, tomando en consideración que dichas acciones aportan al uso de tecnologías en el entorno educativo (Gallo, 2020).

El rendimiento académico esta direccionado a las aulas de clases y la relación docente y estudiante (Lamas, 2015), como consecuencia, necesita utilizar una metodología participativa. Álvarez et al. (2015) establecen que por medio del estudiante se obtiene un rendimiento académico, además, es un valor neto de atributos cognitivos y no cognitivos. Entendiendo así que las nuevas generaciones presentan comportamientos que sumados a las actitudes representan un desafío para el docente (Lara, 2016).

Por su parte, Tordesillas (2019) especifica que la revolución digital se está acelerando a un ritmo sin precedentes, presentando nuevas formas de comunicarse, hacer negocios y aprender. La educación es otro sector que está viendo nuevas tecnologías que ingresan y redefinen el aula cada año. El sector tiene un papel interesante en la revolución digital, porque será responsable de educar a las generaciones actuales y futuras para que utilicen y mejoren la tecnología que respalda la economía digital. Desde los nuevos modelos de enseñanza de la alfabetización, hasta 
el futuro de la educación superior, aquí hay una instantánea de la revolución digital en varios niveles de escolaridad (Cacheiro et al., 2016). Por lo mismo, Ahora se dispone de una amplia gama de recursos pedagógicos como resultado de la revolución digital (Mora et al., 2018).

La transformación digital implica la mejora de los procesos comerciales centrales de una empresa para cumplir de manera efectiva las expectativas del cliente a través del aprovechamiento de datos y tecnología. En el sector educativo, los estudiantes, docentes, personal y graduados pueden ser el consumidor objetivo y tanto los estudiantes, como los docentes, pueden beneficiarse de la transformación digital (Pérez, 2014). Los avances de la tecnología han permitido que la transformación digital llegue a todos los sectores productivos, incluido el educativo y sus integrantes. Esta transformación está vinculada a las tecnologías emergentes, la digitalización de procesos y recursos, y la demanda de los usuarios de actualizarse a las últimas tendencias tecnológicas (Lara et al., 2018). Loureiro et al. (2016) sostienen que la transformación digital del aula va más allá del uso de herramientas y softwares; esta impacta los conocimientos y habilidades de sus usuarios, docentes y estudiantes, y la didáctica y los procesos de las instituciones educativas.

Las habilidades requeridas para la educación y el trabajo han estado sujetas a cambios junto con las tecnologías. La alfabetización digital surgió al mismo tiempo que la evolución de Internet y requería saber cómo acceder, buscar y analizar críticamente la información (Cortés, 2016), por ello, el objetivo del estudio fue proponer estrategias de transformación digital para fortalecer el desempeño docente para establecer beneficios de su uso en los procesos educativos. En tal sentido, es necesario la transformación educativa, debido a que el rol del docente es relevante, ya que pone en foco de evolución en las nuevas metodologías para la enseñanza, el empoderamiento del conocimiento y la aplicación de la tecnología.

\section{Metodología}

Se utilizó un enfoque mixto, por el propósito de estudio se utilizó una investigación aplicada, la que permitió responder al objetivo planteado, lo cual dio a conocer la realidad con evidencia. Además, el alcance fue descriptivo, debido a que se describen fenómenos, hechos y situaciones para ser analizados, del mismo modo, fue explicativo, por lo cual, se pudieron detallar las situaciones generadas. El diseño de investigación fue no experimental y transversal, considerando que la toma de datos fue en un solo momento dado.

La población estuvo conformada por 312 miembros de la Universidad Técnica de Babahoyo (15 docentes y 297 estudiantes). Bajo un muestreo no probabilístico por conveniencia resultaron 172 personas que formaron parte de la investigación entre estudiantes (164) y docentes (9) de la carrera de la institución de educación superior (IES), quienes cumplieron con el criterio de inclusión, el cual fue pertenecer a la IES caso de estudio.

Se propuso una estrategia de transformación digital mediante procesos que permitan diagnosticar el estado actual del desempeño docente, identificar los factores influyentes y diseñar estrategias de transformación digital que ayuden a fortalecer el desempeño docente. La principal potencialidad de la investigación radicó en que los sujetos de la investigación son los actores sociales 
(estudiantes y docentes) de la carrera, que propiciaron los resultados con los que se pueda inferir y generalizar para toda la universidad.

Para la recolección de datos se aplicó una encuesta de treinta ítems, para conocer sobre la variable de estrategias de transformación digital se direcciono ítems relacionados a Internet, era de información, recursos tecnológicos, innovación el aprendizaje hibrido, aprendizaje colaborativo, metodología activa y transformación educativa, unificando un resultado global para conocer el nivel de uso entre bajo, medio y alto. Por otra parte, para lo relacionado con el desempeño docente se tomó en cuenta las habilidades de enseñanza, curiosidad e investigación, desenvolvimiento escénico y comunicación, aprendizaje creativo, creación de un ambiente propicio para el aprendizaje, dominio de dinámicas, educación participativa, rendimiento académico, la aplicación de estrategia, empleo de las TIC, manejo de grupo y logros de objetivos, del mismo modo, a la variable anterior se trabajó con un nivel global de bajo, medio y alto.

Para el procesamiento de información se utilizó el software estadístico SPSS versión 25, en donde se analizaron los datos recabados y se aplicó la prueba Chi Cuadrado como método estadístico, para conocer la relación entre las estrategias de transformación digital y el desempeño del docente.

\section{Resultados}

Del análisis de los datos entre la variable de estrategias de transformación digital y desempeño docente en la carrera de educación básica de la Universidad Técnica de Babahoyo; se obtuvo los valores que se detallan y registran en las tablas a continuación.

Los resultados mostraron que los sujetos que conforman la unidad de investigación, referente a la variable estrategias de transformación digital en la dimensión tecnología fue del 93, 89\%, quienes consideraron que fortalece el uso de la tecnología como Internet, la era digital de información; y los recursos tecnológicos facilitan el proceso de aprendizaje educativo, el desarrollo de conocimientos, las competencias y habilidades.

En la dimensión de innovación; el 91,08\% consideraron que a través del aprendizaje hibrido, el colaborativo y la metodología activa, se facilita el aprendizaje por medio de la innovación, brindando a los estudiantes una educación adecuada y acorde a sus expectativas.

Por otra parte, en la dimensión de trasformación educativa, el 93,99\% considera que la transformación educativa a través del liderazgo, la preparación profesional y la innovación tecnológica, lleva a conseguir objetivos comunes de los estudiantes y el fortalecimiento de los procesos educativos.

En contraste, para la variable de desempeño del docente, los resultados fueron positivos, en la dimensión de habilidades de enseñanza, el 96\% consideró que las habilidades de enseñanza, la curiosidad e investigación, el desenvolvimiento escénico y comunicación y aprendizaje creativo contribuye a la mejora de la práctica educativa. La dimensión de creación de ambientes propicios para el aprendizaje demostró que el 96\% toma en consideración a las habilidades de enseñanza, 
la curiosidad e investigación, el desenvolvimiento escénico, comunicación y aprendizaje creativo para mejorar la practica educativa. Finalmente, el 97,87\% considera que, con la aplicación de estrategia mediante el empleo de las TIC, el manejo de grupo contribuye al logro de objetivos mediante la aplicación de metodologías, lo que a su vez genera nuevas capacidades, esto se evidenció en la dimensión de aplicación de estrategias.

Tabla 1.

Nivel de uso de estrategias de transformación digital y desempeño docente

\begin{tabular}{|c|c|c|c|c|c|}
\hline \multicolumn{2}{|c|}{ BAJO } & \multicolumn{3}{|c|}{ Desempeño Docente } & \multirow[t]{2}{*}{ Total } \\
\hline & & MEDIO & ALTO & & \\
\hline \multirow{6}{*}{$\begin{array}{l}\text { Transformación } \\
\text { Digital }\end{array}$} & BAJO & 1 & 1 & 0 & 2 \\
\hline & & $0,60 \%$ & $0,60 \%$ & $0,00 \%$ & $1,20 \%$ \\
\hline & MEDIO & 0 & 66 & 24 & 90 \\
\hline & & $0,00 \%$ & $38,40 \%$ & $14,00 \%$ & $52,30 \%$ \\
\hline & ALTO & 0 & 21 & 59 & 80 \\
\hline & & $0,00 \%$ & $12,20 \%$ & $34,30 \%$ & $46,50 \%$ \\
\hline \multicolumn{2}{|l|}{ Total } & 1 & 88 & 83 & 172 \\
\hline \multicolumn{2}{|l|}{$0,60 \%$} & $51,20 \%$ & $48,30 \%$ & $100,00 \%$ & \\
\hline
\end{tabular}

Del cruce de variables transformación digital frente al desempeño docente en la carrera de Educación Básica de la Universidad Técnica de Babahoyo, se observó una distribución de frecuencias donde se evidencia con una fuerza de relación de nivel alto con un 48,30\%, medio $51,20 \%$ y bajo $0,06 \%$. Esta información generada permite hacer una inferencia en que existe una relación directa entre las variables observadas.

Tabla 2.

Prueba de correlación del Chi Cuadrado para relación de variables

\begin{tabular}{lccc}
\hline \multicolumn{4}{c}{ Pruebas de chi-cuadrado } \\
\hline Valor & Df & $\begin{array}{c}\text { Significación asintótica } \\
\text { (bilateral) }\end{array}$ \\
\hline Chi-cuadrado de Pearson & $123.785^{\mathrm{a}}$ & 4 &, 000 \\
\hline Razón de verosimilitud & 49.937 & 4 &, 000 \\
\hline Asociación lineal por lineal & 42.505 & 1 &, 000 \\
\hline $\mathrm{N}$ de casos válidos & 172 & & \\
\hline
\end{tabular}

De la relación de las variables, mediante el estadifico Chi-cuadrado de Pearson, se obtuvo un valor de significancia de 0,000 (Tabla 2). Como el valor de sig., fue de 0.000 se afirma que la transformación digital como estrategia se relaciona significativamente con el desempeño del docente. 


\section{Conclusiones}

En la investigación se determinó que las estrategias de transformación digital, a través del uso de tecnologías de la era digital, aportan al aprendizaje y su proceso, estos aspectos que se encuentran relacionados de forma significa con el desempeño del docente, facilitando en cierta forma el desarrollo de competencias, el proceso educativo, y su mejora en el flujo de información. Se obtiene así, una contribución al planteamiento de una propuesta como intervención al uso de la tecnología y por consecuencia al fortalecimiento y mejora del desempeño docente.

Además, la revolución tecnológica desde la innovación facilita el uso de herramientas tecnológicas y, por lo tanto, ayuda a los procesos para el aprendizaje presencial y virtual, aprendizaje colaborativo y aplicación de metodologías activas, que se relacionan de forma significativa con el desempeño de docentes; sin embargo, se requiere involucrar ambientes propicios para el aprendizaje, aplicar procesos y actividades académicas eficientes.

Los resultados de la investigación son el soporte para la aplicación y ejecución de estrategias de transformación digital para que el desempeño del docente sea el adecuado, para ello se promueve la implementación de asesorías personalizadas de forma virtual, con el fin de remover dudas presentes; además de fomentar el contenido virtual, con estos puntos, los estudiantes pueden generar practicas más útiles para el aprendizaje con aplicación de contenidos mediados por las TIC; generando una cultura digital con la que se puede romper acciones habituales, abriéndose al beneficio de las herramientas tecnológicas. 


\section{Referencias}

Álvarez, M., Morales, C., Hernández, D., Cruz, L., \& Cervigni, M. (2015). Predictores cognitivos de rendimiento académico en estudiantes de diseño industrial. Revista Científica de Arquitectura y Urbanismo, 36(1), 86-91. http://scielo.sld.cu/pdf/au/v36n1/au07115.pdf

Bonilla, D., Noboa, G., Ruiz, K., \& Cabrera, J. (2020). Academia, gobierno y empresas una perspectiva desde la vinculacion con la colectividad. Revista de Investigación Enlace Universitario, 19(2), 60-71. https://doi. org/10.33789/enlace.19.2.74

Cacheiro, M., Sánchez, C., \& González, J. (2016). Recursos tecnológicos en contextos la pedagogía contra frente al desaliento educativo. Acción pedagógica, (25), 134-136. http://www.saber.ula.ve/bitstream/handle/123456789/43210/resena1.pdf?sequence=1\&isAllowed=y

Cortés, A. (2016). Prácticas innovadoras de integración educativa de TIC que posibilitan el desarrollo profesional docente. [Tesis doctoral, Universitat Autónoma de Barcelona]. https://www.tdx.cat/bitstream/handle/10803/400225/acr1de1.pdf?sequence $=1$

Escribano, E. (2018). El desempeño del docente como factor asociado a la calidad educativa en América Latina. Revista Educación, 42(2), 717-739. https://doi.org/10.15517/revedu.v42i2.27033

Folch, T., Eudave, D., \& Brunet, I. (2018). Habilidades didácticas de los profesores y creatividad en la educación superior. Perfiles Educativos, 40(162), 100-116. https://doi.org/10.22201/iisue.24486167e.2018.162.58886

Gallo, M. (2020). Análisis exploratorio de los factores de transformación digital en las organizaciones educativas. [Tesis de maestría, Universidad Nacional Abierta y a Distancia]. Repositorio UNAD. https://repository. unad.edu.co/handle/10596/36807

Lamas, H. (2015). Sobre el rendimiento escolar. Revista Propósitos y Representaciones, 3(1), 313-386. https:// doi.org/10.20511/pyr2015.v3n1.74

Lara, A. (2016). Desarrollo y aplicación de una estrategia didáctica para la integración del conocimiento a la enseñanza de la física en ingeniería. Innovación Educativa, 16(71), 133-155. http://www.scielo.org.mx/pdf/ ie/v16n71/1665-2673-ie-16-71-00133.pdf

Lara, A., Lara, N., Lara, G., \& Bonilla, D. (2018). Aprendizaje basado en problemas como estrategia significativa en la formación académica. Universidad, Ciencia y Tecnología, 142-149. http://scielo.sld.cu/pdf/rc/ v14n62/rc066218.pdf

Loureiro, S., Míguez, M., \& Otegui, X. (2016). Desempeño docente en la enseñanza universitaria: análisis de las opiniones estudiantiles. Cuadernos de Investigación Educativa, 7(1), 55. https://doi.org/10.18861/ cied.2016.7.1.2576 
Martínez, G., Guevara, A., \& Valles, M. (2016). El desempeño docente y la calidad educativa. Ra Ximhai, 12(6), 123-134. https://doi.org/10.35197/rx.12.01.e3.2016.06.gm

Mora, E., Bonilla, D., Nuñez, A., \& Sarmiento, C. (2018). Inadaptabilidad de los docentes al manejo de plataformas virtuales: Caso EDUCARECUADOR. Revista Conrado, 14(62), 39-43. http://scielo.sld.cu/pdf/rc/ v14n62/rc066218.pdf

Pérez, I. (2014). La metodología participativa en la educación superior: una evaluación de los estudiantes de sociología. Cuaderno Venezolano de Sociología, 23(4), 643-660. https://www.redalyc.org/ pdf/122/12232821005.pdf

Sánchez, C. (2020). Las redes sociales y las habilidades de enseñanza - aprendizaje del siglo XXI. Eduser, 7(1), 49-55. https://doi.org/10.18050/eduser.v7i1.2511

Tordesillas, C. (2019). El impacto de la revolución digital en la interrelación entre instituciones financieras y sus clientes. Revista icade, (105). https://doi.org/10.14422/icade.i105.y2018.005

Zamora, W., Cobos, D., \& López, F. (2017). Condiciones sociales y salud laboral del profesorado nicaraguense de secundaria. Revista de Pedagogía, 38(103), 192-208. https://www.redalyc.org/pdf/659/65954978010. pdf 
Copyright (c) 2021 Maya Aracely Sánchez Soto, Gina Lorena Camacho Tovar, Edgar Stalyn Guerrero Haro y Cruz Maribel Galarza Ramírez

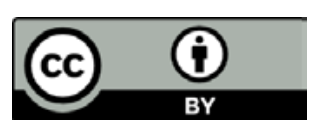

Este texto está protegido bajo una licencia internacional Creative Commons 4.0.

Usted es libre para Compartir-copiar y redistribuir el material en cualquier medio o formato - y Adaptar el documento - remezclar, transformar y crear a partir del material-para cualquier propósito, incluso para fines comerciales, siempre que cumpla las condiciones de Atribución. Usted debe dar crédito a la obra original de manera adecuada, proporcionar un enlace a la licencia, e indicar si se han realizado cambios. Puede hacerlo en cualquier forma razonable, pero no de forma tal que sugiera que tiene el apoyo del licenciante o lo recibe por el uso que hace de la obra.

\section{$\underline{\text { Resumen de licencia - Texto completo de la licencia }}$}

